\title{
Characterizing at-Risk Voxels by Using Perfusion Magnetic Resonance Imaging for Cervical Cancer during Radiotherapy
}

Zhibin Huang ${ }^{1 *}$, Nina A Mayr², Simon S Lo ${ }^{3}$, John C Grecula ${ }^{4}$, Jian Z Wang², Guang Jia ${ }^{4}$ and William TC Yuh ${ }^{4}$

${ }^{1}$ Department of Radiation Oncology, East Carolina University, Greenville, NC 27834, USA

${ }^{2}$ Department of Radiation Oncology, The Ohio State University, Columbus, OH 43210, USA

${ }^{3}$ Department of Radiation Oncology, Case Western Reserve University, Cleveland, OH 44106, USA

${ }^{4}$ Department of Radiology, The Ohio State University, Columbus, OH 43210, USA

\begin{abstract}
The number of voxels with low signal intensity (Low DCE voxels) might be potentially related to treatment failure, which might be associated with the tumor oxygenation status. Our goal was to investigate whether at-risk voxels can be used to predict treatment outcome during radiation therapy for cervical cancer.

80 patients with Stage IB2-IVB cervical cancer were included. Four sequential MRI scans were performed at pre-RT, every 2-2.5 weeks during RT, and post-radiotherapy. 3D volumetric data including tumor regression and tumor perfusion from dynamic contrast enhanced MRI (DCE-MRI) were analyzed. Based on the signal intensity (SI) curves of the DCE-MRI, the low-DCE tumor voxels was obtained for individual patients. The predictive power of low DCE voxels in predicting the treatment outcomes was evaluated by Kaplan-Meier survival analysis. Correlation of Iow DCE voxels with hemoglobin $(\mathrm{Hgb})$ was checked by Pearson Correlation.

The actuarial local control rate and survival rate in the patient group with a small number of low DCE voxels were $89.7 \%$ and $76.9 \%$, compared with $75.6 \%$ and $51.2 \%$ in the patient group with a big number of low DCE voxels for the MRI study $\# 1$, and $94.1 \%$ and $80.4 \%$ compared with $62.1 \%$ and $34.5 \%$ for the MRI study \#2, and $95.7 \%$ and $78.7 \%$ compared with $63.6 \%$ and $42.4 \%$ for the MRI study \#3, respectively. Low DCE voxels were significantly correlated with $\mathrm{Hgb}$.
\end{abstract}

At-risk voxels can be used to predict the outcomes and help understand tumor heterogeneity of response to $\mathrm{RT}$. The Hgb level and tumor perfusion during RT influence the radioresponsiveness and survival in cervical cancer patients.

Keywords: DCE-MRI; Cervical cancer; Radiation therapy; At-risk voxel; Threshold; Signal intensity

\section{Introduction}

It is well-known that tumors are heterogeneous on a microscopic level [1]. Tumor heterogeneity indicates a feature of neoplasia that can precede the tumor itself and reflects variations of tumor cells, which is influenced by both gene mutations and epigenetic gene product regulation. Evaluation of tumor microenvironments has shown heterogeneity relating to hypoxia [2], variation in tumor responsiveness to treatment [3], and gene expression [4]. Many studies have been focused on intratumor heterogeneity across the entire volume of tumors, which is quantified and analyzed in association with clinical outcome for cervical cancer [4-7] and breast cancer [8]. Using DCE-MRI technique, heterogeneous blood perfusion was observed in tumors and variation in blood perfusion may occur within a short time interval [9]. Using PET imaging, among sub-regions with different biological properties within the same tumor is expected to observe tumor heterogeneity or heterogeneous therapy response [10]. Therefore, tumor heterogeneity is a fundamental challenge for personalized cancer care.

Imaging has been introduced in assessing tumor response to radiation and quantitative image biomarkers are of importance in the evaluation of tumor heterogeneity and vascular microenvironment. Different imaging modalities including magnetic resonance imaging (MRI) and positron emission tomography (PET) are used to study on this topic. Compared to PET, MRI doesn't offer high level of biological specificity available with PET, but does provide a flexible imaging modality, as well as a wide range of physiologically relevant image contrasts. Dynamic contrast-enhanced (DCE) MRI has been widely applied in assessing tumor response to radiotherapy. For instance, simplified measurements of change in signal intensity (SI) were investigated, in which the shape of the signal-time course curve and the rate of increase in SI are characterized [6,11-13]. T1-weighted DCEMRI has been applied in early-phase clinical trials, distinguishing the effect of blood volume, blood flow, and contrast agent leakage. Nearly all of DCE-MRI-based studies of antiangiogenic [14-19] and vascular targeting agents [20-22] have utilized T1-weighted DCE-MRI. Early washout of contrast agent has also been strongly related to malignancy [23]. DCE-MRI-derived microcirculation variables including amplitude and the exchange rate constant can be used to evaluate the change in microcirculation $[13,24,25]$.

DCE voxel histogram was first developed to analyze tumor heterogeneity [26], providing identification and quantification of the low DCE voxels at risk of treatment failure within a heterogeneous

*Corresponding authors: Zhibin Huang, Ph.D, Assistant Professor, Departmen of Radiation Oncology, Brody School of Medicine, East Carolina University, 600 Moye Boulevard, Greenville, NC 27834, USA, Tel: 252-744-3768; Fax: 252-7443775; E-mail: huangz@ecu.edu

Received January 13, 2012; Accepted August 11, 2012; Published August 13 2012

Citation: Huang Z, Mayr NA, Lo SS, Grecula JC, Wang JZ, et al. (2012) Characterizing at-Risk Voxels by Using Perfusion Magnetic Resonance Imaging for Cervical Cancer during Radiotherapy. J Cancer Sci Ther 4: 254-259. doi:10.4172/1948-5956.1000151

Copyright: $\odot 2012$ Huang Z, et al. This is an open-access article distributed unde the terms of the Creative Commons Attribution License, which permits unrestricted use, distribution, and reproduction in any medium, provided the original author and source are credited. 
tumor. It has been demonstrated that the heterogeneity DCE parameter could be used to predict outcomes within 2-2.5 weeks during radiation therapy (RT) [26]. However, it is necessary to further investigate this method with the increase of patient population and to explore the predictive power of low DCE voxels.

We have characterized the threshold value of signal intensity (SI) percentile [27] that was defined as SI accumulated in critically low DCE voxels within the subregions of the heterogeneous tumor, at different time points of treatment. Low DCE voxels might have the potential to further improve the established paradigm for monitoring tumor response and predicting outcomes in early phase of treatment.

The purpose of this study was to 1) determine the low DCE voxels within the heterogeneous tumor using the threshold of SI in the three MRI studies; 2) investigate the predictive power of the low DCE voxels by correlating the number of DCE voxels with clinical outcomes; 3 ) investigate the correlation between the low DCE voxels and hemoglobin level.

\section{Materials and Methods}

\section{Patient characteristics}

Serial MRI including 3D tumor volume and DCE tumor perfusion imaging, were performed in 80 cancer patients. Patient characteristics are presented in Table 1. Pre-treatment evaluations included routine work-up following FIGO guidelines [28]. Therapy consisted of standard combined external beam RT with 6 to $24 \mathrm{MeV}$ photons beams, concurrent weekly cisplatin-based chemotherapy, and standard low dose rate brachytherapy. The external beam RT dose to the pelvis ranged from 39.6 to $66.6 \mathrm{~Gy}$ (mean $47.8 \mathrm{~Gy}$ ) including field reductions at 1.8 to $2.0 \mathrm{~Gy} /$ fraction. All but 4 patients had brachytherapy, with Point A doses ranging from 14 to $61 \mathrm{~Gy}$ (mean $39.5 \mathrm{~Gy}$ ).

\section{Assessment of longitudinal hemoglobin (Hgb) levels}

Longitudinal $\mathrm{Hgb}$ levels were assessed routinely before and during the RT course at weekly intervals. In this study, the Hgb levels were calculated as the pre-RT Hgb, assessed $\leq 10$ days before the start of RT, the lowest Hgb level before and/or during therapy (nadir Hgb), and the mean $\mathrm{Hgb}(\mathrm{mHgb})$, the mean of all $\mathrm{Hgb}$ levels before and during the RT course, including external beam RT and brachytherapy.

\section{DCE-MRI acquisition}

The serial MRI scans were performed prospectively at 4 well-defined time points: at start of RT (MRI \#1), twice during RT including early RT (at 20-25 Gy of pelvic RT) (MRI \#2) and mid-way RT (at 40-50 Gy) (MRI \#3), and at follow-up (1-2 months after completion all RT including brachytherapy) (MRI \#4). All of the 80 patients completed all 4 MRI scans. The MRI scans were obtained with 1.5 Tesla superconductive scanners using a body coil. Imaging included sagittal 5-mm fast spin echo T2-weighted images $\left(\mathrm{TE}_{\text {eff }}=104 \mathrm{~s}, \mathrm{TR}=4000 \mathrm{~s}, \mathrm{ETL}=10, \mathrm{NEX}=2\right)$ and axial 7-mm T2-weighted and T1-weighted images ( $\mathrm{TE}=16 \mathrm{~s}, \mathrm{TR}=600$ $s, \mathrm{NEX}=2$ ). A single bolus injection of a paramagnetic contrast agent (Gd-DTPA, dose $0.1 \mathrm{mmol} / \mathrm{kg} @ 4 \mathrm{ml} / \mathrm{s}$ ) produces a transient signal enhancement on a T1-weighted sensitive imaging sequence.

\section{Image analysis}

In each MRI scan, the tumor region was delineated as region of interest (ROI) based on the T2-weighted image by two independent examiners-a radiation oncologist and a gynecologic oncologist. ROIs were transferred to the DCE T1-weighted images. The tumor was delineated on each T2-weighted imaging slice and the anatomical 3D tumor volume
(ATV), was derived by summation of all the tumor voxels within the region-of-interest tumor region based on the tumor delineation from the T2-weighted images as described previously $[26,29,30]$.

The first-pass DCE method [31] as described in the DCE imaging was used to obtain a time-signal intensity (SI) DCE curve generated for each tumor voxel. Our group first developed tumor voxel SI histogram method, in which voxel histograms were derived from each tumor based upon total tumor voxels for each of the three DCE-MRI studies representing different time points [32]. Based on the SI distribution of the voxel SI histogram, tumor heterogeneity can be readily displayed and appreciated by the variation and wide range of SI values at individual voxels within the entire tumor.

\section{Low DCE voxels (number of voxels with low intensity)}

Low DCE voxels represent the voxels with a low SI value. The SI over low DCE voxels was summed up to achieve the specific SI value (e.g. $\mathrm{SI}=2.1$, here we call it a threshold value) to extract the number of low DCE voxels, which might be associated with the tumor heterogeneity. Tumor response to RT varies with the degree of the heterogeneity; therefore, the number of low DCE voxels is different from tumor to tumor in order to achieve the same signal intensity.

Thresholds of SI values were almost the same $(=2.1)$ for the 3 series MRI scans. Therefore, the number of low DCE voxels was characterized by summing up the SI of low DCE voxels to achieve the signal intensity equal to the threshold of SI value.

\section{Treatment end points}

Patients were followed regularly post-therapy in 3-6 monthly intervals. Local control was defined as no tumor recurrence or persistence within pelvis during the follow-up period. For diseasespecific survival analysis, death due to causes other than cervical cancer was considered a censored observation.

\section{Statistical analysis}

All statistical analyses were performed on the software (SPSS 17, Spss Inc. Chicago, IL). Correlations between at-risk voxels and the clinical outcome were assessed by either the Mann-Whiteney rank sum test or Pearson correlation. Receiver operating characteristics (ROC) analysis was adopted to select optimal model classifiers. Survival curves were obtained using Kaplan-Meier survival analysis.

\section{Results}

\section{Image analysis}

The contours were digitized and input into in-house MATLAB

\begin{tabular}{|c|c|}
\hline & Patients $(n=80)$ \\
\hline Age (years) & $55(25.0-89.0)$ \\
\hline \multicolumn{2}{|l|}{ FIGO stage } \\
\hline I & $10(12 \%)$ \\
\hline II & $31(39 \%)$ \\
\hline III & $28(35 \%)$ \\
\hline IV & $11(14 \%)$ \\
\hline \multicolumn{2}{|l|}{ Histology } \\
\hline Squamous cell ca. & $69(86 \%)$ \\
\hline Adenocarcinoma & $11(14 \%)$ \\
\hline \multicolumn{2}{|l|}{ Brachytherapy } \\
\hline Low-dose-rate & $79(99 \%)$ \\
\hline High-dose-rate & $1(1 \%)$ \\
\hline Chemotherapy & $37(47 \%)$ \\
\hline
\end{tabular}


software. Figure 1 showed the contour was determined on the T2wieghted image and then was automatically transferred to the T1weighted images using in-house MATLAB software for calculating the SI and the number of voxels.

SI values at individual voxels and the number of voxels were obtained, resulting in a distribution of SI values at individual voxels in the ROI. A temporal change of mean SI of DCE-MRI for a typical patient normally includes a baseline observed with a low SI value. After the contrast agent was injected, the SI increased dramatically and reached a maximum value in plateau phase for typical patients (from $90 \mathrm{~s}$ to about $115 \mathrm{~s})$.

\section{Number of low DCE voxels}

In this study, the threshold value of SI was selected to be 2.1. To achieve this signal intensity, the number of low DCE voxels that reflects the heterogeneity of tumor was determined for 80 patients. Histogram of low DCE voxels for 80 patients showed that the number of low DCE voxels varied from patient to patient, and indicated the degree to which the tumor responds to RT.

\section{Correlation of low DCE voxels with Hgb levels}

Hgb levels were significantly correlated with the low DCE voxel in the first MRI study ( $p=0.001$, Pearson Correlation), and correlated significantly with the low DCE voxels in the second MRI study ( $\mathrm{p}=0.002$, Pearson Correlation), and continued to correlate significantly with the low DCE voxels in the third MRI study ( $\mathrm{p}=0.024$, Pearson Correlation).

\section{Correlation of low DCE voxels with treatment outcome}

Figure 2 showed the ROC analysis based on disease specific survival, which was used to determine an optimal threshold for the number of low DCE voxels at each MRI study. The area under curve (AUC) and threshold values of the number of low DCE voxels were determined for MRI scan \#1, \#2, and \#3, respectively, as shown in Table 2. Using each of threshold values, the Kaplan-Meier survival analysis was performed. Table 2 showed the correlation of the number of at-risk voxels with treatment outcome, including the p value from the KaplanMeier survival analysis for 80 patients in the MRI scan \#1, \#2 and \#3, respectively.

Figure 3 showed the Kaplan-Meier analysis of local control at different time points: MRI \#1 at the beginning of RT, MRI \#2 at 2-2.5 weeks of RT, MRI \#3 at 1-2 months after RT completion. The 6-year actuarial local control rate in the patient group with the small number of low DCE voxels $(<311)$ was $89.7 \%$ compared with $75.6 \%$ in the patient group with the big number of low DCE voxels $(\geq 311 ; \mathrm{p}=0.045$, log-rank test) for the MRI study \#1. The 6-year actuarial local control rate in the patient group with the small number of low DCE voxels $(<247)$ was $94.1 \%$ compared with $62.1 \%$ in the patient group with the big number of low DCE voxels ( $\geq 247$; $p<0.0001$, log-rank test) for the MRI study \#2. The 6-year actuarial local control rate in the patient group with the small number of low DCE voxels $(<123)$ was $95.7 \%$ compared with $63.6 \%$ in the patient group with the big number of low DCE voxels $(\geq 123 ; \mathrm{p}<0.0001, \log$-rank test) for the MRI \#3.

Figure 4 showed Kaplan-Meier analysis of disease specific survival at different time points: MRI \# 1 at the beginning of RT, MRI \#2 at 2-2.5 weeks of RT, MRI \#3 at 1-2 months after RT completion. The 6-year disease-specific survival rate was $76.9 \%$ in the patient group with the small number of low DCE voxels $(<311)$ compared with $51.2 \%$ in the patient group with the big number of low DCE voxels $(\geq 311 ; p=0.025$, log-rank test) for the MRI \#1. The 6-year disease-specific survival rate was $80.4 \%$ in the patient group with the small number of low DCE voxels $(<247)$ compared with $34.5 \%$ in the patient group with the big number of low DCE voxels ( $\geq 247$; $p<0.0001, \log$-rank test) for the MRI \#2. The 6-year disease-specific survival rate was $78.7 \%$ in the patient group with the small number of low DCE voxels $(<123)$ compared with $42.4 \%$ in the patient group with the big number of low DCE voxels ( $\geq 123$; $\mathrm{p}<0.001, \log$-rank test) for the MRI \#3.

\section{Multivariate analysis}

Multivariate analysis including tumor stage, lymph node status, hemoglobin level and the low DCE voxels showed that the number of low DCE voxels is the most significant variable in predicting outcomes (Hazard Ratio: 7.88, Confidential Interval: 1.66-37.36, p=0.009).

\section{Discussion}

We have been collecting DCE-MRI perfusion data for cervical cancer since 1993. The perfusion data from a single-center prospective clinical trial ensure consistence in imaging quality. DCE-MRI can be used to analyze both morphology and kinetic characteristics of tumors. Despite the availability of functional imaging techniques for assessing tumor perfusion and hypoxia, tumor imaging has not traditionally applied to target functional tumor heterogeneity and define its unfavourable component that likely contributes to the treatment failure. For many tumors including cervical cancer, currently the established clinical prognostic criteria and treatment response assessment have been traditionally based upon FIGO staging and the morphologybased tumor volume measurement.

In this study, we focused on the predictive power of at-risk voxels, although tumor volume is a prognostic factor in predicting clinical outcome [33]. The parameter of at-risk voxels is able to reflect the intra-patient and inter-patient response to radiation because the more at-risk voxels in a tumor, the more resistant the tumor response is and the more the heterogeneity is in a tumor. Using DCE-MRI technique, heterogeneous tumor perfusion was observed and variations in blood perfusion may occur within a short time interval [9].

Criteria to characterize tumor heterogeneity and define high-risk tumor voxels with unfavorable functional properties within tumor subregions, which translates into poor clinical outcome, are lacking. In this study, we analyzed DCE-MRI perfusion data and provided a novel,

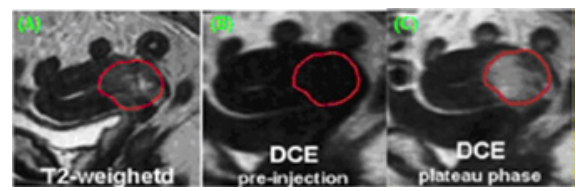

Figure 1: Contour drawn in $T_{2}$-weighted image $(A)$ and transferred to $T_{1}$ weighted image before contrast injection $(B)$ and after contrast injection in plateau phase $(\mathrm{C})$.
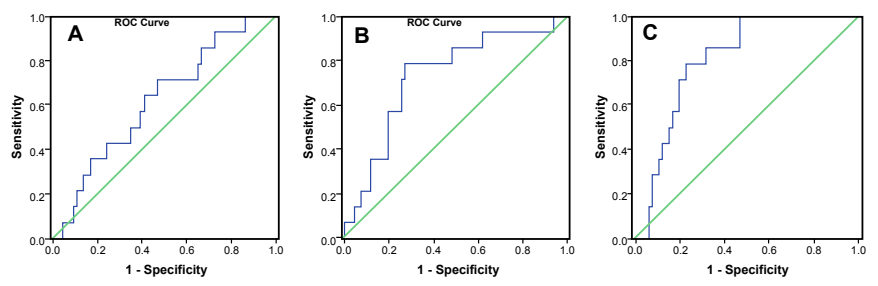

Figure 2: ROC curves of number of at-risk voxels in the MRI study \#1, \#2, and \#3 for LC with SI value equal to 2.1 . 

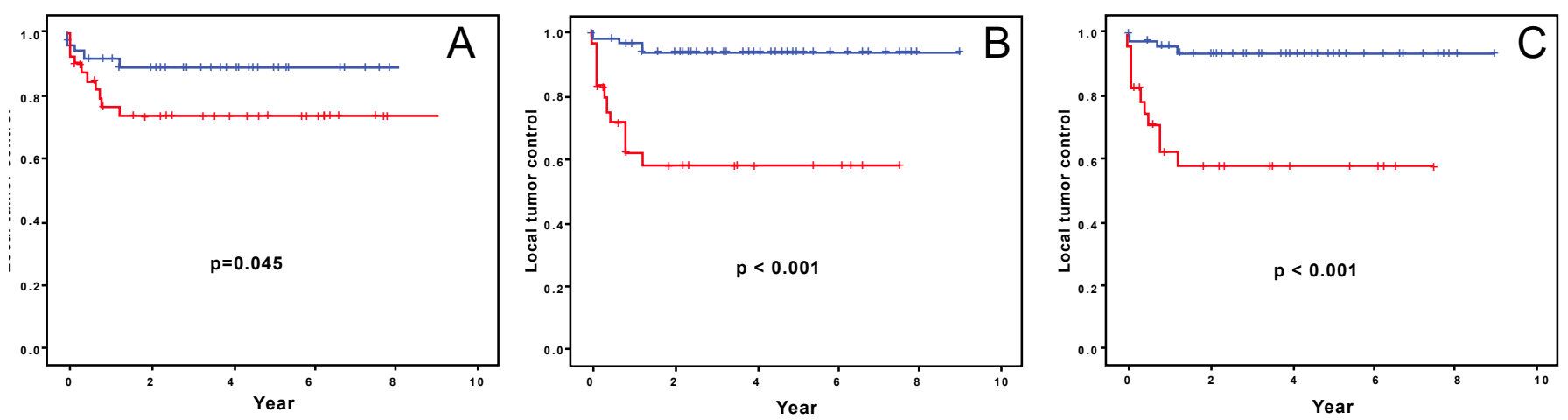

Figure 3: Kaplan-Meier survival curves for the number of at-risk voxels for LC in the MRI study A) \#1, B) \#2, and C) \#3.
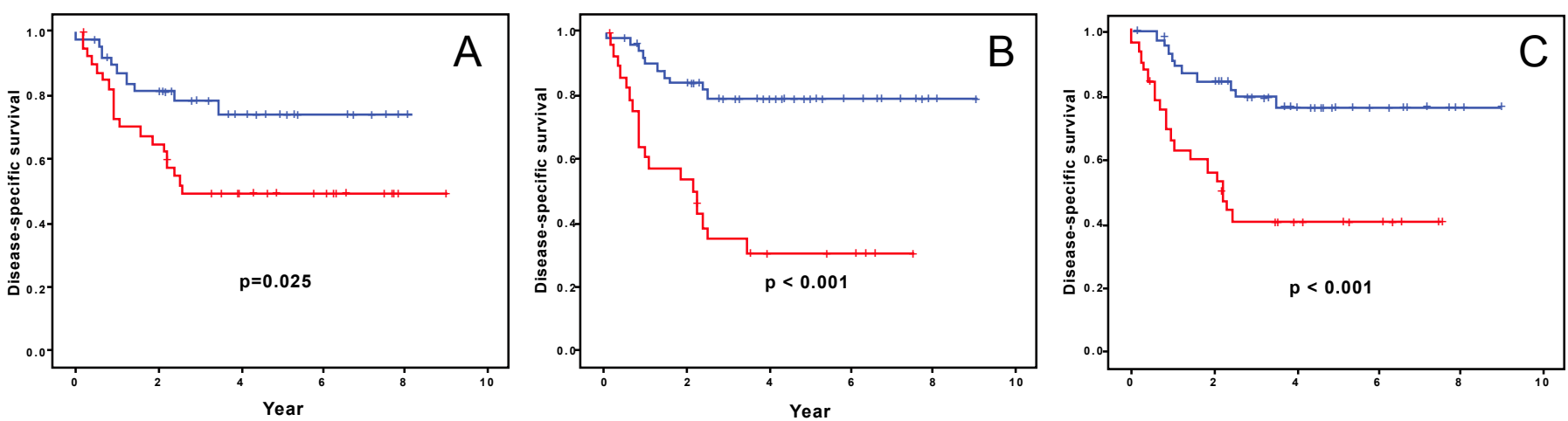

Figure 4: Kaplan-Meier survival curves for the number of at-risk voxels for DSS in the MRI study A) \#1, B) \#2, and C) \#3.

\begin{tabular}{|c|c|c|c|c|c|c|}
\hline \multirow[b]{2}{*}{ MRI Study } & \multicolumn{3}{|c|}{ LC } & \multicolumn{3}{|c|}{ DSS } \\
\hline & \#1 & \#2 & \#3 & $\# 1$ & \#2 & \#3 \\
\hline AUC & 0.620 & 0.729 & 0.807 & 0.661 & 0.711 & 0.707 \\
\hline threshold & 311 & 247 & 123 & 311 & 247 & 123 \\
\hline $\begin{array}{l}\text { Below threshold } \\
\text { vs. } \\
\text { Above threshold }\end{array}$ & $\begin{array}{l}89.7 \% \\
\text { vs. } \\
75.6 \%\end{array}$ & $\begin{array}{l}94.1 \% \\
\text { vs. } \\
62.1 \%\end{array}$ & $\begin{array}{c}95.7 \% \\
\text { vs. } \\
63.6 \%\end{array}$ & $\begin{array}{c}76.9 \% \\
\text { vs. } \\
51.2 \%\end{array}$ & $\begin{array}{c}80.4 \% \\
\text { vs. } \\
34.5 \%\end{array}$ & $\begin{array}{l}78.7 \% \\
\text { vs. } \\
42.4 \%\end{array}$ \\
\hline $\mathrm{p}$ value (Kaplan-Meier) & 0.045 & 7.7E-5 & $7.1 \mathrm{E}-5$ & 0.025 & $1.1 \mathrm{E}-5$ & 3.7E-4 \\
\hline
\end{tabular}

Table 2: Correlation of the number of at-risk voxels with treatment outcome.

non-invasive means to characterize functional heterogeneity and the parameter: the number of the low DCE (at-risk) voxels that might be an important prognostic factor for clinical outcome using DCE-MRI. The histogram of the number of low DCE voxels reflects the extent to which the tumors respond to RT.

The SI optimal values used to characterize at-risk voxels are of importance. The ROC analysis provides an optimal classifier such that SI value was selected to be 2.1 in this study, based on the area under the ROC curve (AUC), as shown in Figure 2. Our study showed SI=2.1 was a reasonable optimal value to characterize treatment failure. Using another concept of SI percentile, previous studies determined SI percentile $10 \%$ to be the best cutoff for separating patients in association with clinical outcome [34]. Coincidently, the threshold value of SI percentile $10 \%$ was exactly 2.1 . Apparently it would be easier to understand the tumor heterogeneity when we used the parameterthe number of low DCE voxels involved in achieving a certain amount of SI.

Characterizing the at-risk voxels for treatment failure provides a means to investigate tumor heterogeneity in sub-regions of an entire tumor. A voxel-SI distribution histogram analysis method [35] was first developed to identify, separate and analyze each tumor voxel's functional imaging biomarker within the heterogeneous tumor. Based upon the clinically validated optimal SI threshold values at various treatment times, the poorly perfused at-risk tumor voxels among the entire tumor can be identified for differentiation of poor treatment outcome from those favorable ones.

At-risk voxels were significantly correlated with the Hgb levels for the three MRI studies. Our results suggest that the oxygenation may play an important role in the formation of tumor heterogeneity. Further investigation on the combined effect of at-risk voxels and the Hgb levels would facilitate understanding the relationship between tumor heterogeneity and oxygenation.

It is expected that tumor heterogeneity or heterogeneous therapy response exists among sub-regions with different biological properties within the same tumor [10]. This gives rise to a fundamental challenge 
Citation: Huang Z, Mayr NA, Lo SS, Grecula JC, Wang JZ, et al. (2012) Characterizing at-Risk Voxels by Using Perfusion Magnetic Resonance Imaging for Cervical Cancer during Radiotherapy. J Cancer Sci Ther 4: 254-259. doi:10.4172/1948-5956.1000151

for personalized cancer care. Therefore, it is necessary to have high resolution anatomic and functional imaging before and during treatment, which allows serial risk assessment based upon the therapyspecific changes of tumor voxels' DCE values at various treatment time points. Most treatment failures in cervical cancer occur within 2 years. Our patient population, which is the largest with the longest follow-up time (mean: 6.8 years) reported to date, provided a solid data base for statistical analysis and clinical validation of the predictive power of atrisk tumor voxels for treatment failure.

\section{Conclusion}

Our data analysis demonstrated that the number of at-risk voxels is a prognostic factor in predicting treatment outcomes and helps understand tumor heterogeneity in response to radiation therapy. The results suggest that the Hgb level and tumor perfusion during $\mathrm{RT}$ influence the radioresponsiveness and survival in cervical cancer patients.

\section{Statement of Translational Relevance}

Heterogeneity of tumor response to radiation therapy plays an important role in cancer therapy. This study provides an approach to characterizing tumor heterogeneity in response to radiation. At-risk voxels identified by using DCE-MRI has been found for predicting clinical outcomes in cervical cancer during radiation therapy. Once the DCE parameter indicates the possible failure of the radiation scheme for patients, an aggressive radiation plan during radiation course would be adopted for salvage.

\section{Acknowledgment}

The work was supported in part by the National Institutes of Health under contract [R01 CA 71906]

\section{References}

1. Heppner GH (1984) Tumor heterogeneity. Cancer Res 44: 2259-2265.

2. Hockel M, Schlenger K, Aral B, Mitze M, Schaffer U, et al. (1996) Association between tumor hypoxia and malignant progression in advanced cancer of the uterine cervix. Cancer Res 56: 4509-4515.

3. Britten RA, Evans AJ, Allalunis-Turner MJ, Franko AJ, Pearcey RG (1996) Intratumoral heterogeneity as a confounding factor in clonogenic assays for tumour radioresponsiveness. Radiother Oncol 39: 145-153.

4. Bachtiary B, Boutros PC, Pintilie M, Shi W, Bastianutto C, et al. (2006) Gene expression profiling in cervical cancer: an exploration of intratumor heterogeneity. Clin Cancer Res 12: 5632-5640.

5. Jain RK, Baxter LT (1988) Mechanisms of heterogeneous distribution of monoclonal antibodies and other macromolecules in tumors: significance of elevated interstitial pressure. Cancer Res 48: 7022-7032.

6. Jackson A, O'Connor JP, Parker GJ, Jayson GC (2007) Imaging tumor vascular heterogeneity and angiogenesis using dynamic contrast-enhanced magnetic resonance imaging. Clin Cancer Res 13: 3449-3459.

7. Kidd EA, Grigsby PW (2008) Intratumoral metabolic heterogeneity of cervica cancer. Clin Cancer Res 14: 5236-5241.

8. Han A, Yang L, Frazier AB (2007) Quantification of the heterogeneity in breast cancer cell lines using whole-cell impedance spectroscopy. Clin Cancer Res 13: $139-143$

9. Brurberg KG, Benjaminsen IC, Dørum LM, Rofstad EK (2007) Fluctuations in tumor blood perfusion assessed by dynamic contrast-enhanced MRI. Magn Reson Med 58: 473-481.

10. Zhao S, Kuge Y, Mochizuki T, Takahashi T, Nakada K, et al. (2005) Biologic correlates of intratumoral heterogeneity in 18F-FDG distribution with regional expression of glucose transporters and hexokinase-II in experimental tumor. $\mathrm{J}$ Nucl Med 46: 675-682

11. Turetschek K, Preda A, Novikov V, Brasch RC, Weinmann HJ, et al. (2004) Tumor microvascular changes in antiangiogenic treatment: assessment by magnetic resonance contrast media of different molecular weights. J Magn Reson Imaging 20: 138-144.

12. Preda A, Novikov V, Möglich M, Floyd E, Turetschek K, et al. (2005) Magnetic resonance characterization of tumor microvessels in experimental breast tumors using a slow clearance blood pool contrast agent (carboxymethyldextran-A2Gd-DOTA) with histopathological correlation. Eur Radiol 15: 2268-2275.

13. Hillengass J, Wasser K, Delorme S, Kiessling F, Zechmann C, et al. (2007) Lumbar bone marrow microcirculation measurements from dynamic contrastenhanced magnetic resonance imaging is a predictor of event-free survival in progressive multiple myeloma. Clin Cancer Res 13: 475-481.

14. Jayson GC, Zweit J, Jackson A, Mulatero C, Julyan P, et al. (2002) Molecular imaging and biological evaluation of HuMV833 anti-VEGF antibody: implications for trial design of antiangiogenic antibodies. J Natl Cancer Inst 94: 1484-1493.

15. Morgan B, Thomas AL, Drevs J, Hennig J, Buchert M, et al. (2003) Dynamic contrast-enhanced magnetic resonance imaging as a biomarker for the pharmacological response of PTK787/ZK 222584, an inhibitor of the vascular endothelial growth factor receptor tyrosine kinases, in patients with advanced colorectal cancer and liver metastases: results from two phase I studies. J Clin Oncol 21: 3955-3964.

16. O'Donnell A, Padhani A, Hayes C, Kakkar AJ, Leach M, et al. (2005) A Phase I study of the angiogenesis inhibitor SU5416 (semaxanib) in solid tumours, incorporating dynamic contrast MR pharmacodynamic end points. $\mathrm{Br} \mathrm{J}$ Cancer 93: 876-883.

17. Thomas AL, Morgan B, Horsfield MA, Higginson A, Kay A, et al. (2005) Phase I study of the safety, tolerability, pharmacokinetics, and pharmacodynamics of PTK787/ZK 222584 administered twice daily in patients with advanced cancer J Clin Oncol 23: 4162-4171.

18. Wedam SB, Low JA, Yang SX, Chow CK, Choyke P, et al. (2006) Antiangiogenic and antitumor effects of bevacizumab in patients with inflammatory and locally advanced breast cancer. J Clin Oncol 24: 769-777.

19. Mullamitha SA, Ton NC, Parker GJ, Jackson A, Julyan PJ, et al. (2007) Phase I evaluation of a fully human anti-alphav integrin monoclonal antibody (CNTO 95 ) in patients with advanced solid tumors. Clin Cancer Res 13: 2128-2135.

20. Dowlati A, Robertson K, Cooney M, Petros WP, Stratford M, et al. (2002) A phase I pharmacokinetic and translational study of the novel vascular targeting agent combretastatin a-4 phosphate on a single-dose intravenous schedule in patients with advanced cancer. Cancer Res 62: 3408-3416.

21. Galbraith SM, Maxwell RJ, Lodge MA, Tozer GM, Wilson J, et al. (2003) Combretastatin A4 phosphate has tumor antivascular activity in rat and man as demonstrated by dynamic magnetic resonance imaging. $\mathrm{J}$ Clin Oncol 21 : 2831-2842.

22. McKeage MJ, Fong $P$, Jeffery M, Baguley BC, Kestell P, et al. (2006) 5,6-Dimethylxanthenone-4-acetic acid in the treatment of refractory tumors: a phase I safety study of a vascular disrupting agent. Clin Cancer Res 12: 17761784 .

23. Kuhl CK, Mielcareck P, Klaschik S, Leutner C, Wardelmann E, et al. (1999) Dynamic breast MR imaging: are signal intensity time course data useful for differential diagnosis of enhancing lesions? Radiology 211: 101-110.

24. Knopp MV, Weiss E, Sinn HP, Mattern J, Junkermann H, et al. (1999) Pathophysiologic basis of contrast enhancement in breast tumors. J Magn Reson Imaging 10: 260-266.

25. Nosàs-Garcia S, Moehler T, Wasser K, Kiessling F, Bartl R, et al. (2005) Dynamic contrast-enhanced MRI for assessing the disease activity of multiple myeloma: a comparative study with histology and clinical markers. J Magn Reson Imaging 22: 154-162.

26. Mayr NA, Tali ET, Yuh WT, Brown BP, Wen BC, et al. (1993) Cervical cancer: application of MR imaging in radiation therapy. Radiology 189: 601-608.

27. Mayr NA, Yuh WT, Jajoura D, Wang JZ, Lo SS, et al. (2010) Ultra-early predictive assay for treatment failure using functional magnetic resonance imaging and clinical prognostic parameters in cervical cancer. Cancer 116: 903-912.

28. (1988) Annual Report of Treatment in Gynecologic Cancer. Int Fed Gynecol Obstet 20: 40

29. Mayr NA, Yuh WT, Zheng J, Ehrhardt JC, Sorosky JI, et al. (1997) Tumor size evaluated by pelvic examination compared with $3-D$ quantitative analysis in the prediction of outcome for cervical cancer. Int J Radiat Oncol Biol Phys 39: 395-404.

30. Mayr NA, Taoka T, Yuh WT, Denning LM, Zhen WK, et al. (2002) Method and timing of tumor volume measurement for outcome prediction in cervical cance using magnetic resonance imaging. Int J Radiat Oncol Biol Phys 52: 14-22. 
Citation: Huang Z, Mayr NA, Lo SS, Grecula JC, Wang JZ, et al. (2012) Characterizing at-Risk Voxels by Using Perfusion Magnetic Resonance Imaging for Cervical Cancer during Radiotherapy. J Cancer Sci Ther 4: 254-259. doi:10.4172/1948-5956.1000151

31. Yuh WT (1999) An exciting and challenging role for the advanced contrast MR imaging. J Magn Reson Imaging 10: 221-222.

32. Mayr NA, Yuh WT, Arnholt JC, Ehrhardt JC, Sorosky JI, et al. (2000) Pixel analysis of MR perfusion imaging in predicting radiation therapy outcome in cervical cancer. J Magn Reson Imaging 12: 1027-1033.

33. Mayr NA, Magnotta VA, Ehrhardt JC, Wheeler JA, Sorosky JI, et al. (1996) Usefulness of tumor volumetry by magnetic resonance imaging in assessing response to radiation therapy in carcinoma of the uterine cervix. Int $\mathrm{J}$ Radiat Oncol Biol Phys 35: 915-924.
34. Yuh WT, Mayr NA, Jarjoura D, Wu D, Grecula JC, et al. (2009) Predicting control of primary tumor and survival by DCE MRI during early therapy in cervical cancer. Invest Radiol 44: 343-350.

35. Mayr NA, Yuh WT, Magnotta VA, Ehrhardt JC, Wheeler JA, et al. (1996) Tumor perfusion studies using fast magnetic resonance imaging technique in advanced cervical cancer: a new noninvasive predictive assay. Int $\mathrm{J}$ Radia Oncol Biol Phys 3: 623-633. 